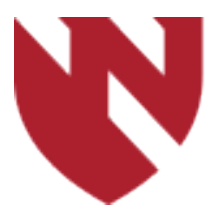

September 2020

\title{
Endoscopic removal of Fractured 3D-Printed Nasopharyngeal Swabs from the Olfactory Cleft During COVID-19
}

\author{
Matthew Miller \\ University of Nebraska Medical Center \\ Kristy Carlson \\ University of Nebraska Medical Center \\ Jayme Dowdall \\ University of Nebraska Medical Center \\ Christie Barnes \\ University of Nebraska Medical Center \\ Sam Pate \\ University of Nebraska Medical Center
}

Tell us how you used this information in this short survey.

Follow this and additional works at: https://digitalcommons.unmc.edu/gmerj

Part of the Anatomy Commons, Higher Education Commons, Otolaryngology Commons, and the Virus Diseases Commons

\section{Recommended Citation}

Miller, M, Carlson, K, Dowdall, J, \& Pate, S. Endoscopic removal of Fractured 3D-Printed Nasopharyngeal Swabs from the Olfactory Cleft During COVID-19.

This Case Report is brought to you for free and open access by DigitalCommons@UNMC. It has been accepted for inclusion in Graduate Medical Education Research Journal by an authorized editor of DigitalCommons@UNMC. For more information, please contact digitalcommons@unmc.edu. 


\title{
Endoscopic removal of Fractured 3D-Printed Nasopharyngeal Swabs from the Olfactory Cleft During COVID-19
}

\author{
Abstract \\ The severe acute respiratory syndrome SARS-CoV-2 pandemic has posed significant challenges to \\ hospital and public health systems in the United States. In response to a shortage in nasopharyngeal \\ swabs, our institution developed, tested, and deployed 3D-printed swabs to obtain a specimen for testing. \\ A patient was tested in preparation for a scheduled Esophagogastrodeuodenoscopy. During the \\ procedure the swab broke and a portion remained in the superior meatus and olfactory cleft. This case \\ study describes the process of locating and removing the broken sections of the 3D-printed swab. In \\ response to this incident, 3D-printing teams test swabs for sample collection and rheology with an \\ elongation test. Additionally, we have implemented just-in-time training for staff including point-of-care \\ durability testing, model simulation with feedback to ensure accurate trajectory, and suggestions on how \\ to address anatomic and other challenges.
}

\section{Keywords}

COVID-19, Coronavirus, SARS-CoV-2, Test, 3D printing, Nasopharyngeal Swab, 3D Printing, Retained foreign body

\section{Creative Commons License} c) (i) $(\Theta$

This work is licensed under a Creative Commons Attribution-Noncommercial-No Derivative Works 4.0 License.

\section{Cover Page Footnote}

We would like to thank our colleagues in 3D printing, specifically Mr. Trent Allen at Tethon 3D, virology testing, infectious disease and nursing for their contributions to patient care and diagnostic testing during this pandemic and also for their critical review of the manuscript. 


\section{Endoscopic Removal of Fractured 3D-Printed Nasopharyngeal Swabs From the Olfactory Cleft During COVID-19}

Matthew Miller ${ }^{1}$, Kristy Carlson ${ }^{1}$, Jayme Dowdall ${ }^{1}$, Christie Barnes ${ }^{1}$, Sam Pate ${ }^{1}$

${ }^{1}$ University of Nebraska Medical Center, Department of Otolaryngology - Head \& Neck Surgery

\begin{abstract}
The severe acute respiratory syndrome SARS-CoV-2 pandemic has posed significant challenges to hospital and public health systems in the United States. In response to a shortage in nasopharyngeal swabs, our institution developed, tested, and deployed 3D-printed swabs to obtain a specimen for testing. A patient was tested in preparation for a scheduled Esophagogastrodeuodenoscopy. During the procedure, the swab broke and a portion remained in the superior meatus and olfactory cleft. This case study describes the process of locating and removing the broken sections of the 3D-printed swab. In response to this incident, 3D-printing teams initiated testing the swabs used for sample collection and rheology with an elongation test. Additionally, we have implemented justin-time training for staff, including point-ofcare durability testing, model simulation with feedback to ensure accurate trajectory, and suggestions on how to address anatomic and other challenges.
\end{abstract}

\section{Introduction}

The severe acute respiratory syndrome coronavirus 2 (SARS-CoV-2) pandemic and the disease it causes, COVID-19, has posed significant challenges to hospital and public health systems in the United States. At the current time, RT-PCR is the primary means of testing. The timing and location of specimen collection for SARS-CoV-2 play an important role in diagnosis.1,2and is sustained by severe acute respiratory syndrome coronavirus 2 (SARS-CoV-2 Similar to other sites across the country, our institution has experienced supply chain challenges, including obtaining swabs for specimen collection.3 This scarcity in resources has resulted in increased innovation, including the production of 3D-printed nasopharyngeal (NP) swabs.

Our institution developed, tested, and deployed 3D-printed swabs, starting in early April 2020. Here we present a case of a fractured NP swab requiring endoscopic retrieval. We will discuss design implications as institutions across the country take to similar measures to address the NP swab shortage.

\section{Case}

The NP swabs used were 3D printed, manufactured in sections, cured together and sterilized via Sterrad (Figure 1A).

An inpatient was scheduled for Esophagogastrodeuodenoscopy (EGD) for melena with positive fecal occult blood test. Because EGD is a high risk/aerosol generating procedure (AGP), COVID-19 testing was performed. During the swab of the right nasal cavity, the patient reported significant discomfort. When the swab was pulled out of the nose, only $1 / 2-2 / 3$ length of the swab was removed (Figure $1 \mathrm{~B})$.

On exam with nasal speculum and headlight, a dobhoff tube was noted in the left nasal cavity and secured with a bridle. The patient had a right septal deflection and mild turbinate hypertrophy. The pathway along the nasal floor therefore was quite narrow. No retained swab was appreciated, prompting right nasal endoscopy and retrieval. Appropriate COVID-19 PPE for an APG was used.

Decongestion and anesthesia were achieved via Afrin and 4\% lidocaine on cotton pledgets placed bilaterally. Nasal endoscopy confirmed septal deflection and turbinate hypertrophy, making access to the nasopharynx along the floor of the nose difficult. The nasopharynx was accessed endoscopically along the top of the inferior turbinate under the free edge of the middle turbinate. A minimal amount of blood was identified in the posterior $2 / 3$ of the nasal cavity, which was suctioned along with mucus. There were no swab remnants visualized in the nasopharynx nor the middle meatus.

The endoscope was then inserted medial to the middle turbinate, with visualization of the superior meatus and olfactory cleft. Remnants of the broken swab were found in this area. Using an alligator forceps, a $1 \mathrm{~cm}$ swab remnant was retrieved from the olfactory cleft. Reconstruction of the broken swab revealed that a portion of the swab was still missing. More thorough evaluation into the superior meatus allowed visualization of the remainder of the swab, which was removed with alligator forceps. The patient tolerated this well and experienced immediate relief.

\section{Discussion}

Due to the scarcity of NP swabs, 3D-printing is a method used by a number of institutions to combat the supply chain challenges. In response to this incident, we have partnered closely with 3D-printing teams who are responsible for testing the swabs used for sample collection and rheology with an elongation test. We have implemented justin-time training for staff, including point-ofcare durability testing, model simulation with feedback to ensure accurate trajectory, and suggestions on how to address anatomic and other challenges.

To our knowledge, there is no specific, published testing protocol for NP swab prototypes. We present a case of a patient with challenging anatomy, obstructing medical devices (NG tubes, bridles) all of which are commonplace in the inpatient setting. We recommend caution in such patients as this may direct the swab superiorly. In addition to a nasal examination, we recommend visual inspection of the swab prior to inserting into the sample tube to verify full extraction.

https://doi.org/10.32873/unmc.dc.gmerj.2.1.071

\section{References}

1 Pang J, Wang MX, Ang IYH, et al. Potential Rapid Diagnostics, Vaccine and Therapeutics for 2019 Novel Coronavirus (2019-nCoV): A Systematic Review. Journal of Clinical Medicine. 2020;9(3):623. doi: $10.3390 / \mathrm{jcm} 9030623$

2 Lippi G, Simundic A-M, Plebani M. Potential preanalytical and analytical vulnerabilities in the laboratory diagnosis of coronavirus disease 2019 (COVID-19). Clin Chem Lab Med. March 2020. doi:10.1515/cclm-2020-0285

3 Shortage of COVID-19 sample collection tools will be short-lived, suppliers say. Modern Healthcare. https:// www.modernhealthcare.com/supply-chain/shortagecovid-19-sample-collection-tools-will-be-short-livedsuppliers-say. Published March 22, 2020. Accessed April 15, 2020 


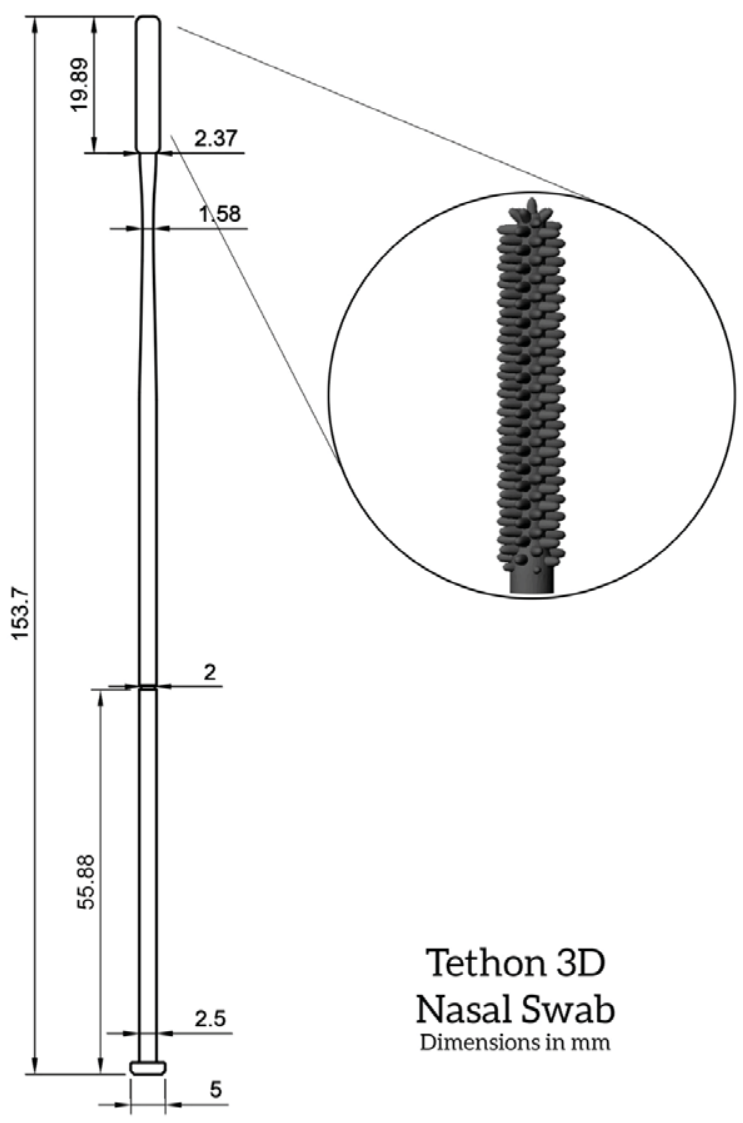

$1 \mathrm{~A}$.

Figure 1A. Dimensions of the 3D printed swab.

Figure 1B. A side-by-side comparison of a nasopharyngeal swab and the two sections removed from the patient. 